\title{
HOLOMORPHIC MAPPINGS INTO TEICHMÜLLER SPACES
}

\author{
HARUMI TANIGAWA \\ (Communicated by Clifford J. Earle, Jr.)
}

\begin{abstract}
Let $T(F)$ be the Teichmüller space of a finitely generated Fuchsian group $F$ of the first kind. We shall show that there exists no nontrivial family of holomorphic proper mappings of the unit disc into $T(F)$ with a complex analytic parameter. Using this result, we shall investigate a family of quasi-conformal mappings with a complex analytic parameter whose Beltrami differentials vanish on a fixed set of positive area.
\end{abstract}

\section{INTRODUCTION}

Let $F$ be a finitely generated Fuchsian group of the first kind. The Teichmüller space $T(F)$ of $F$ is identified, via the Bers embedding, with a bounded domain in $\mathrm{C}^{m}$, where $m$ is the dimension of $T(F)$. Hence a holomorphic mapping of the unit disc $\Delta$ into $T(F)$ is regarded as a finite system of bounded holomorphic functions on $\Delta$. Shiga [Sh1, Sh2] applied theorems on bounded holomorphic functions to such a mapping to prove several results on Teichmüller spaces. On the other hand, a lot of properties of $T(F)$ and its boundary have been pointed out by many researchers. Some of these results, for example Lemmas $1,2,3$ in $\S 3$, indicate that the boundary is complicated. With these properties, the Fatou's theorem and the Riesz' theorem about bounded analytic functions, Imayosi-Shiga [IS] gave an analytic proof of the finiteness theorem of holomorphic families over Riemann surfaces of finite type.

In this paper, we also utilize the properties of the boundary of $T(F)$ and theorems on bounded analytic functions to show certain rigidity properties of holomorphic proper mappings of the unit disk $\Delta$ into $T(F)$ (actually, we deal with holomorphic mappings under some weaker condition). Namely, there exists no nontrivial family of holomorphic proper mappings of $\Delta$ into $T(F)$ with a complex analytic parameter. As a corollary we shall show that $T(F)$ is not holomorphically equivalent to a product of complex manifolds. We shall also investigate, as a corollary, some families of quasi-conformal mappings of Riemann surfaces.

\section{Notation}

In this section we fix our notation and recall some known results. We refer to Kra [K2] and Lehto [L] for more details and references.

Received by the editors July 1, 1990 and, in revised form, May 2, 1991.

1991 Mathematics Subject Classification. Primary 32G15; Secondary 30F10. 
Throughout this paper, $\Delta$ denotes the unit disk and $H$ denotes the upper half plane and $L$ denotes the lower half plane. In this paper, a quasi-conformal mapping is a quasi-conformal homeomorphism of the Riemann sphere $\widehat{C}$. Let $F$ be a finitely generated Fuchsian group of the first kind acting on the upper half plane $H . F$ is said to be of signature $\left(p, n ; \alpha_{1}, \ldots, \alpha_{n}\right)$ (or simply, $(p, n)$ ) if the Riemann surface $H / F$ has genus $p$ and has $n$ special points with ramification number $\alpha_{1}, \ldots, \alpha_{n}$. Here we consider a puncture of $H / F$ as a special point of ramification number $\infty$. An essentially bounded measurable function on $\widehat{C}$ satisfying

$$
\mu \circ g\left(\overline{g^{\prime}} / g^{\prime}\right)=\mu \text { a.e. }
$$

for all $g \in F$ is called a Beltrami differential for $F$.

Let $B(F)$ denote the space of Beltrami differentials for $F$. The space $B(F)$ is a Banach space with supremum norm. Let $B_{1}(F)$ denote the unit ball of $B(F)$. For $F$-invariant set $U, B(F, U)$ denotes the space of Beltrami differentials for $F$ that vanish outside of $U$ and $B_{1}(F, U)$ denotes the unit ball of $B(F, U)$. For each $\mu \in B_{1}(F), w^{\mu}$ denotes the quasi-conformal homeomorphism of $\widehat{C}$ with Beltrami differential $\mu$ normalized so that $w^{\mu}$ fixes 0,1 , and $\infty$.

A quasi-conformal homeomorphism $w$ is called compatible with $F$ provided

$$
w \circ g \circ w^{-1} \in \operatorname{PSL}(2, C)
$$

for all $g \in F$. A quasi-conformal homeomorphism $w$ is compatible with $F$ if and only if its Beltrami differential belongs to $B_{1}(F)$. Two Beltrami differentials $\mu_{1}$ and $\mu_{2}$ are called equivalent if $w^{\mu_{1}}$ and $w^{\mu_{2}}$ induce the same group isomorphism of $F$ into $\operatorname{PSL}(2, C)$; that is,

$$
w^{\mu_{1}} \circ g \circ\left(w^{\mu_{1}}\right)^{-1}=w^{\mu_{2}} \circ g \circ\left(w^{\mu_{2}}\right)^{-1}
$$

for all $g \in F$. The Teichmüller space $T(F)$ of the Fuchsian group $F$ is the set of all equivalence classes that are determined by elements of $B_{1}(F, H)$. Let $\mu_{1}$ and $\mu_{2}$ be elements of $B_{1}(F, H)$. Set

$$
\hat{\mu}_{i}(z)=\left\{\begin{array}{ll}
\mu_{i}(z) & z \in H, \\
\bar{\mu}_{i}(\bar{z}) & z \in L,
\end{array} \quad i=1,2 .\right.
$$

The Beltrami differentials $\mu_{1}$ and $\mu_{2}$ determine the same group isomorphism if and only if $\hat{\mu}_{1}$ and $\hat{\mu}_{2}$ determine the same group isomorphism.

Now we introduce the Bers embedding of $T(F)$ into $C^{3 p-3+n}$. Let $A_{2}(F, L)$ be the space of holomorphic functions on the lower half plane $L$ satisfying

$$
\phi \circ g \times\left(g^{\prime}\right)^{2}=\phi \quad \forall g \in F
$$

and

$$
\sup \left\{\left|(\operatorname{Im}(z))^{2} \phi(z)\right| ; z \in L\right\}<\infty .
$$

The space $A_{2}(F, L)$ is a $(3 p-3+n)$-dimensional complex linear space, hence equivalent to $C^{3 p-3+n}$. Set

$$
\widehat{\boldsymbol{\Phi}}(\mu)=S_{w^{\mu} \mid L}
$$

where $\mu \in B_{1}(F, H)$ and $S_{w^{\mu} \mid L}$ stands for the Schwarzian derivative of $w^{\mu} \mid L$. Then $\widehat{\Phi}(\mu)$ depends only on the equivalence class $[\mu] \in T(F)$ and $\widehat{\Phi}(\mu)$ belongs to $A_{2}(F, L)$. Thus the mapping $\widehat{\Phi}: B_{1}(F, H) \rightarrow A_{2}(F, L)$ induces a 
mapping $\Phi: T(F) \rightarrow A_{2}(F, L)$. The mapping $\Phi$ is a holomorphic injection and gives an embedding (called the Bers embedding) of $T(F)$ onto a bounded domain of $A_{2}(F, H)$. We identify $T(F)$ and $\Phi(T(F))$, and regard $T(F)$ as a bounded domain in $C^{3 p-3+n}$.

We describe the boundary $\partial T(F)$ of $T(F)$ briefly (see Bers [B1], Maskit [M] for details). For each element $\phi \in \bar{T}(F)$ there exists a univalent function $W_{\phi}$ of the lower half plane $L$ such that $S_{W}=\phi$ and that

$$
W_{\phi}(z)=(z+i)^{-1}+O(|z+i|), \quad \text { near } z=-i .
$$

Since $\phi \in \bar{T}(F) \subset A_{2}(F, L)$,

$$
S_{W \circ g}=S_{W}=\phi,
$$

for each element $g$ of $F$. Hence there exists an element $\theta_{\phi}(g)$ of $\operatorname{PSL}(2, C)$ such that

$$
\theta_{\phi}(g) \circ W_{\phi}=W_{\phi} \circ g .
$$

The correspondence $g \mapsto \theta_{\phi}(g)$ is an isomorphism of $F$ into $\operatorname{PSL}(2, C)$. It is known that if $\phi \in T(F)$ then $\theta_{\phi}(F)$ is a quasi-fuchsian group and that if $\phi \in \partial T(F)$, then $\theta_{\phi}(F)$ is a Kleinian group with exactly one invariant component $W_{\phi}(L)$. The Kleinian group $\theta_{\phi}(F)$ is called a cusp provided there exists a hyperbolic element $g \in F$ such that $\theta_{\phi}(g)$ is parabolic. The Kleinian group $\theta_{\phi}(F)$ with $\phi \in \partial T(F)$ is called a regular b-group if the Poincare area of the Riemann surface $W_{\phi}(L) / \theta_{\phi}(F)$ is half of that of $\Omega\left(\theta_{\phi}(F)\right) / \theta_{\phi}(F)$. The Kleinian group $\theta_{\phi}(F)$ is called a totally degenerate b-group if $W_{\phi}(L)$ is a dense open subset of $\widehat{C}$. It is known that if $\phi \in \partial T(F)$ and $\theta_{\phi}(F)$ is not a cusp, then $\theta_{\phi}(F)$ is a totally degenerate $b$-group.

For a quasi-conformal selfmapping $w$ of $\widehat{C}$ with Beltami differential $\mu$, the maximal dilatation $K(w)$ is given by

$$
K(w)=\frac{1+\|\mu\|_{\infty}}{1-\|\mu\|_{\infty}} .
$$

The Teichmüller distance $d$ on $T(F)$ is defined by

$$
\begin{array}{r}
d\left(\left[\mu_{0}\right],\left[\nu_{0}\right]\right)=\inf \left\{\frac{1}{2} \log K\left(w^{\mu} \circ\left(w^{\nu}\right)^{-1}\right) ; \mu, \nu \in B_{1}(F, H),\right. \\
\left.[\mu]=\left[\mu_{0}\right],[\nu]=\left[\nu_{0}\right]\right\}
\end{array}
$$

where $\left[\mu_{0}\right],\left[\nu_{0}\right] \in T(F)$.

The Kobayashi (or hyperbolic) pseudodistance $d_{M}$ on a complex manifold $M$ is given as follows; for two points $p, q \in M$, choose points $p=p_{0}, p_{1}, \ldots$, $p_{k-1}, p_{k}=q$ of $M$, points $a_{1}, \ldots, a_{k}, b_{1}, \ldots, b_{k}$ of $\Delta$, and holomorphic mappings $f_{1}, \ldots, f_{k}$ of $\Delta$ into $M$ such that $f_{i}\left(a_{i}\right)=p_{i-1}$ and $f_{i}\left(b_{i}\right)=p_{i}$ for $i=1, \ldots, k$. Then

$$
d_{M}(p, q)=\inf \sum_{i=1}^{k} \rho\left(a_{i}, b_{i}\right),
$$

where $\rho$ is the Poincare distance on $\Delta$ and the infimum is taken with respect to all possible choices of finite points and holomorphic mappings as above. A complex manifold is called hyperbolic provided $d_{M}$ is a distance. When $M=\Delta, d_{M}$ coincides with $\rho$. For a finitely generated Fuchsian group of the 
first kind $F$, the Kobayashi pseudodistance $d_{T(F)}$ is a distance and coincides with the Teichmüller distance $d$. Let $M$ and $N$ be two complex manifolds and let $f: M \rightarrow N$ be a holomorphic mapping. Then from the definition of the Kobayashi pseudodistances,

$$
d_{M}(p, q) \geq d_{N}(f(p), f(q)), \quad p, q \in M .
$$

\section{RIGIDITY OF HOLOMORPHIC MAPPINGS INTO TEICHMÜLLER SPACES}

In this section, $F$ denotes a finitely generated Fuchsian group of the first kind whose signature is $(p, n)$ with $3 p-3+n>0$. The Teichmüller space $T(F)$ is identified with a bounded domain in $C^{3 p-3+n}$ via the Bers embedding. Let $\Delta$ be the unit disk $\{z \in C ;|z|<1\}$. We regard a holomorphic mapping $f: \Delta \rightarrow T(F)$ as a bounded holomorphic mapping $\Delta \rightarrow C^{3 p-3+n}$. Hence the mapping $f$ has radial limits at almost all points of $\partial \Delta=\{z \in C ;|z|=1\}$, and the limits belong to $\bar{T}(F)$. We begin with some lemmas.

Lemma 1 (Shiga [Sh1, Theorem 5]). Let $f$ be a holomorphic mapping of $\Delta$ into $T(F)$. Then the set

$$
\begin{array}{r}
E_{\mathrm{cusp}}=\left\{e^{i \theta} \in \partial \Delta ; f \text { has a radial limit } f_{*}\left(e^{i \theta}\right) \text { at } e^{i \theta},\right. \\
\text { which corresponds to a cusp }\}
\end{array}
$$

has linear measure 0.

Lemma 2 (Shiga [Sh2, Theorem 5]). Let $f$ be a holomorphic mapping of $\Delta$ into $\bar{T}(F)$. If $f(0)$ corresponds to a totally degenerate b-group, then the mapping $f$ is a constant mapping, that is, $f \equiv f(0)$ on $\Delta$.

Remark. In fact, the statement of Shiga [Sh2] Theorem 5 is slightly different from that of the above lemma. But the same argument yields the above statement.

Lemma 2 yields another proof of the following lemma.

Lemma 3 (Bers [B3, Lemma 2]). Let $\left\{\varphi_{j}\right\}$ and $\left\{\psi_{j}\right\}$ be sequences in $T(F)$ such that $d\left(\varphi_{j}, \psi_{j}\right)<M \quad(j=1,2, \ldots)$ for a universal constant $M<\infty$. If $\lim _{j \rightarrow \infty} \varphi_{j}=\varphi$ exists and $\varphi$ corresponds to a totally degenerate group, then $\lim _{j \rightarrow \infty} \psi_{j}=\varphi$.

Proof. For each $j$, there exists a holomorphic isometry $\Psi_{j}: \Delta \rightarrow T(F)$ such that $\Psi_{j}(0)=\varphi_{j}$ and $\Psi_{j}(\Delta) \ni \psi_{j}$. Then by the assumption,

$$
\rho\left(0, \Psi_{j}^{-1}\left(\psi_{j}\right)\right)=d\left(\varphi_{j}, \psi_{j}\right)<M .
$$

Here $\rho$ is the Poincare distance on $\Delta$ and $d$ is the Teichmüller distance on $T(F)$. Hence the sequence $\left\{\Psi_{j}^{-1}\left(\psi_{j}\right)\right\}$ is contained in a compact set in $\Delta$. Suppose that there exists a subsequence $\left\{\psi_{j_{k}}\right\}$ of $\left\{\psi_{j}\right\}$ such that $\lim _{k \rightarrow \infty} \psi_{j_{k}} \neq$ $\varphi$. By taking a subsequence if necessary, we may assume that the sequence of mappings $\left\{\Psi_{j_{k}}\right\}$ converges to a holomorphic mapping $\Psi^{0}: \Delta \rightarrow \bar{T}(F)$ and that the sequence $\left\{\Psi_{j_{k}}^{-1}\left(\psi_{j_{k}}\right)\right\}$ converges to a point $z_{0} \in \Delta$. Then $\Psi^{0}(0)=\varphi$, which corresponds to a totally degenerate group, and $\Psi^{0}\left(z_{0}\right) \neq \varphi$. This contradicts Lemma 2. 
Theorem 1. Let $f: \Delta \times \Delta \rightarrow T(F)$ be a holomorphic mapping with the following property: there exists a point $\zeta_{0} \in \Delta$ such that the set

$$
\begin{array}{r}
E_{\zeta_{0}}=\left\{e^{i \theta} \in \partial \Delta ; f\left(\cdot, \zeta_{0}\right) \text { has a radial limit } f_{*}\left(e^{i \theta}, \zeta_{0}\right) \text { at } e^{i \theta}\right. \\
\text { and that } \left.f_{*}\left(e^{i \theta}, \zeta_{0}\right) \in \partial T(F)\right\}
\end{array}
$$

has positive linear measure. Then $f$ depends only on the first variable $z$, i.e., for all $\zeta, \zeta^{\prime} \in \Delta$,

$$
f(\cdot, \zeta) \equiv f\left(\cdot, \zeta^{\prime}\right) \text { on } \Delta .
$$

Proof. By Lemma 1, the measure of the set

$$
E_{\zeta_{0}, \text { cusp }}=\left\{e^{i \theta} \in E_{\zeta_{0}} ; f_{*}\left(e^{i \theta}, \zeta_{0}\right) \text { corresponds to a cusp }\right\}
$$

is 0 . Set $\widehat{E}_{\zeta_{0}}=E_{\zeta_{0}}-E_{\zeta_{0} \text {, cusp }}$. For each point $e^{i \theta} \in \widehat{E}_{\zeta_{0}}$ there exists a point $\phi_{\theta} \in \partial T(F)$ corresponding to a totally degenerate group such that

$$
\lim _{r \rightarrow 1} f\left(r e^{i \theta}, \zeta_{0}\right)=\phi_{\theta} .
$$

Fix a point $\zeta \in \Delta$. Since the holomorphic mapping $f$ does not inciease the hyperbolic distances,

$$
d\left(f\left(r e^{i \theta}, \zeta_{0}\right), f\left(r e^{i \theta}, \zeta\right)\right) \leq d_{\Delta \times \Delta}\left(\left(r e^{i \theta}, \zeta_{0}\right),\left(r e^{i \theta}, \zeta\right)\right)=d_{\Delta}\left(\zeta_{0}, \zeta\right) .
$$

For each increasing sequence $\left\{r_{n}\right\}$ with $0<\cdot r_{n}<1$ and $\lim _{n \rightarrow \infty} r_{n}=1$, there exists a subsequence $\left\{r_{n_{j}}\right\}$ such that the limit $\lim _{n_{j} \rightarrow \infty} f\left(r_{n_{j}} e^{i \theta}, \zeta\right)$ exists. By (3.4) and Lemma 3, the limit is equal to $\phi_{\theta}$. Hence we have

$$
\lim _{r \rightarrow 1} f\left(r e^{i \theta}, \zeta\right)=\phi_{\theta} .
$$

Since the mappings $f(\cdot, \zeta)$ and $f\left(\cdot, \zeta_{0}\right)$ are both bounded and have the same radial limit at each point of the positive measure set $\widehat{E}_{\zeta_{0}}, f(\cdot, \zeta) \equiv f\left(\cdot, \zeta_{0}\right)$ on $\Delta$. Noting that $\zeta$ is an arbitrary point of $\Delta$, the assertion of the theorem follows.

Remark 1. Lemma 2 yields another proof of Theorem 1, without using Kobayashi distance, which we omit here.

Remark 2. In connection with Theorem 1, there arises a natural question that the same statement as Theorem 1 is true for infinite-dimensional Teichmüller spaces. To this question, Professor C. McMullen suggested counterexamples. The details will be given elsewhere.

\section{Corollaries to Theorem 1}

In this section we derive corollaries from Theorem 1 . Let $F$ be a finitely generated Fuchsian group of the first kind. The following is immediate.

Corollary 1. There exists no nontrivial family of proper holomorphic mappings of $\Delta$ into $T(F)$ with a complex analytic parameter.

A holomorphic mapping $\widetilde{\Phi}: \Delta \rightarrow B_{1}(F, H)$ is projected to a holomorphic mapping $\Phi=\pi \circ \widetilde{\Phi}: \Delta \rightarrow T(F)$, here $\pi: B_{1}(F, H) \rightarrow T(F)$ is the canonical projection. The holomorphic mapping $\Phi$ has radial limits in $\bar{T}(F)$ almost everywhere in $\partial \Delta$. Let $\omega$ be a point of $\partial \Delta$ such that the radial limit 
$\Phi_{*}(\omega)=\lim _{r \rightarrow 1} \Phi(r \omega)$ exists. Set $\widetilde{\Phi}(\zeta)=\mu_{\zeta}$ for $\zeta \in \Delta$. If $\Phi_{*}(\omega) \in \partial T(F)$, then $\lim _{r \rightarrow 1}\left\|\mu_{r \omega}\right\|_{\infty}=1$. But of course the converse is not true in general. It is easy to construct a holomorphic mapping $\widetilde{\Phi}: \Delta \rightarrow B_{1}(F, H)$ such that $\lim _{r \rightarrow 1}\left\|\mu_{r \omega}\right\|_{\infty}=1$ for all $\omega \in \partial \Delta$ and that the image $\Phi(\Delta)$ is relatively compact in $T(F)$. We claim that almost all radial limits belong to $T(F)$ provided each Beltrami differential $\mu_{\zeta}=\widetilde{\boldsymbol{\Phi}}(\zeta), \zeta \in \Delta$, vanishes on a fixed nontrivial set.

Corollary 2. Let $F$ be a finitely generated Fuchsian group of the first kind acting on $H$. Suppose that a holomorphic mapping $\widetilde{\Phi}: \Delta \rightarrow B_{1}(F, H)$ has the following property: there exists a $F$-invariant subset $E$ of $H$ with two-dimensional Lebesgue measure positive such that each Beltrami differential $\widetilde{\Phi}(\zeta)=\mu_{\zeta}, \zeta \in$ $\Delta$, vanishes on $E$. Let $\Phi: \Delta \rightarrow T(F)$ be the projection of $\tilde{\Phi}$ on $T(F)$. Then almost all radial limits of $\Phi$ on $\partial \Delta$ are contained in $T(F)$.

Proof. In proving the corollary, we may assume that $\mu_{0}=0$. In fact it is well known (cf. [K2, §3.1]) that there exist a Fuchsian group $F^{\prime}$ and biholomorphic maps $\tilde{g}: B_{1}(F, H) \rightarrow B_{1}\left(F^{\prime}, H\right)$ and $g: T(F) \rightarrow T\left(F^{\prime}\right)$ such that $\tilde{g}\left(\mu_{0}\right)=0$, $\pi^{\prime} \circ \tilde{g}=g \circ \pi$, and $\pi^{\prime} \circ \tilde{g} \circ \widetilde{\Phi}=g \circ \Phi$, where $\pi: B_{1}(F, H) \rightarrow T(F)$ and $\pi^{\prime}: B_{1}\left(F^{\prime}, H\right) \rightarrow T\left(F^{\prime}\right)$ are the natural projections. (Note that $\tilde{g}\left(B_{1}(F, E)=\right.$ $B_{1}\left(F, E^{\prime}\right)$ for some positive measure set $E^{\prime}$ since a quasi-conformal map is absolutely continuous.) To prove the corollary, it is sufficient to show that there exists a Beltrami differential $\nu$ in $B_{1}(F, E)$ such that the holomorphic mapping $\Psi: \Delta \times \Delta \rightarrow T(F)$ defined by

$$
\Psi(z, \zeta)=\left[\mu_{z}+\zeta \nu\right],
$$

depends on the second variable $\zeta$ nontrivially. Since the set $E$ is a positive measure set, $B_{1}(F, E)$ is projected onto a neighborhood of the origin [0] of $T(F)$ under the natural projection $B_{1}(F, E) \rightarrow T(F)$. Hence there exists a Beltrami differential $\nu \in B_{1}(F, E)$ such that $\left[\mu_{0}\right] \neq[\nu / 2]$. Such a Beltrami differential has the desired property.

Corollary 3. Let $F$ be a finitely generated Fuchsian group of the first kind whose signature is $(p, n)$ with $3 p-3+n>0$. Then the Teichmüller space $T(F)$ is not holomorphically equivalent to a direct product $M_{1} \times M_{2}$, where $M_{i}$ is a complex manifold of positive dimension, $i=1,2$.

Proof. Assume that there exist complex manifolds $M_{1}$ and $M_{2}$ and a biholomorphic mapping $\Phi=\left(\Phi_{1}, \Phi_{2}\right)$ of $T(F)$ onto $M_{1} \times M_{2}$, where $\Phi_{i}$ is a holomorphic mapping of $T(F)$ to $M_{i}, i=1,2$. Take a holomorphic proper mapping $h: \Delta \rightarrow T(F)$, and set $E=\left\{e^{i \theta} \in \partial \Delta ; h\right.$ has a radial limit $h_{*}\left(e^{i \theta}\right)$ at $e^{i \theta}$ and that $\left.h_{*}\left(e^{i \theta}\right) \in \partial T(F)\right\}$. For each $e^{i \theta} \in E$, set $c_{\theta}=\left\{r e^{i \theta} ; 0<r<1\right\}$. Then either $\Phi_{1} \circ h\left(c_{\theta}\right)$ is not relatively compact in $M_{1}$ or $\Phi_{2} \circ h\left(c_{\theta}\right)$ is not relatively compact in $M_{2}$. Putting

$$
E_{i}=\left\{e^{i \theta} \in E ; \Phi_{i} \circ h\left(c_{\theta}\right) \text { is not relatively compact in } M_{i}\right\}, \quad i=1,2,
$$

we have $E=E_{1} \cup E_{2}$. Since meas $(E)>0$, we may assume that meas $\left(E_{1}\right)>0$, where meas stands for the linear measure. For each point $p \in M_{2}$, define the holomorphic mapping $\Psi_{p}: \Delta \rightarrow T(F)$ by

$$
\Psi_{p}(z)=\Phi^{-1}\left(\Phi_{1} \circ h(z), p\right), \quad z \in \Delta .
$$


Then the set

$$
E_{1}^{p}=\left\{e^{i \theta} \in E_{1} ; \Psi_{p} \text { has a radial limit } \Psi_{p *}\left(e^{i \theta}\right)\right\}
$$

has the same (hence positive) linear measure as $E_{1}$, and each $e^{i \theta} \in E_{1}^{p}$, $\Psi_{p *}\left(e^{i \theta}\right) \in \partial T(F)$. Note that the family of holomorphic mappings $\left\{\Psi_{p}\right\}_{p \in M_{2}}$ depends holomorphically on the parameter $p$. This contradicts Theorem 1 .

Next we give a remark on holomorphic families over Riemann surfaces of finite type. We begin with a brief description. See for example Imayoshi-Shiga [IS], which contains an analytic proof of Parsin-Arakerov's theorem, for more details and references. Let $F_{1}$ and $F_{2}$ be finitely generated torsion free Fuchsian groups of the first kind acting on $\Delta$, and let $(p, n)$ be the signature of $F_{2}$. A holomorphic mapping $\Phi$ of $\Delta$ into $T\left(F_{2}\right)$ is called a holomorphic family of type $(p, n)$ over the Riemann surface $\Delta / F_{1}$ if for each $g \in F_{1}$ there exists a holomorphic homeomorphism $\chi(g)$ of $T\left(F_{2}\right)$ such that $\Phi \circ g=\chi(g) \circ \Phi$. The correspondence $g \rightarrow \chi(g)$ is a homomorphism. Two holomorphic families $\Phi_{1}$ and $\Phi_{2}$ are called equivalent if there exists a holomorphic homeomorphism $\gamma$ of $T\left(F_{2}\right)$ such that $\Phi_{1}=\gamma \circ \Phi_{2}$. Noguchi [N] investigated the set of holomorphic mappings of certain kinds of complex spaces and showed that the set of holomorphic families over $\Delta / F_{1}$ is a Zariski open subset of a compact complex space, hence consists of finitely many components. If a holomorphic family $\Phi$ is not a constant map, from the argument in [IS] (from p. 212 line 32 to p. 213 line 18), radial limits of $\Phi$ at almost everywhere in $\partial \Delta$ belong to $\partial T(F)$. Hence from Theorem 1 it follows that the component containing $\Phi$ consists of one point. Thus we get another proof of Parshin-Arakelov's finiteness theorem of holomorphic families;

Corollary 4. For given finitely generated torsion free Fuchsian group of the first kind $F_{1}$ and a pair of nonnegative integers $(p, n)$ with $2 p-2+n>0$, there exists only finitely many nonequivalent and locally nontrivial holomorphic families of type $(p, n)$ over $\Delta / F_{1}$.

\section{ACKNOWLEDGMENT}

The author would like to express her thanks to Professor H. Shiga for helpful conversation. She also would like to thank the referee of the present version of this paper for useful comments and the referee of an earlier version for suggestions of the improved version of Corollary 3 that is given here. (The proof of it given in this paper is a simpler one than he suggested.)

\section{REFERENCES}

[AB] L. V. Ahlfors and L. Bers, Riemann's mapping theorem for variable metrics, Ann. of Math. (2) 72 (1960), 385-404.

[B1] L. Bers, On boundaries of Teichmüller spaces and on Kleinian groups. I, Ann. of Math. (2) 91 (1970), 570-600.

[B2] _ Fiber spaces over Teichmüller spaces, Acta Math. 130 (1973), 83-126.

[B3] - On iterates of hyperbolic transformations, Amer. J. Math. 105 (1983), 1-11.

[BR] L. Bers and H. J. Royden, Holomorphic families of injections, Acta. Math. 157 (1986), 159-186. 
[IS] Y. Imayoshi and H. Shiga, A finiteness theorem for holomorphic families of Riemann surfaces, Holomorphic Functions and Moduli II, Springer-Verlag, New York, Heidelberg, and Berlin, 1987, pp. 207-219.

[K1] I. Kra, On spaces of Kleinian groups, Comment. Math. Helv. 47 (1972), 53-69.

[K2] _ Canonical mappings between Teichmüller spaces, Bull. Amer. Math. Soc. (N. S.) 4 (1981), 207-219.

[L] O. Lehto, Univalent functions and Teichmüller spaces, Springer-Verlag, New York, Heidelberg, and Berlin, 1985.

[M] B. Maskit, On boundaries of Teichmüller spaces and on Kleinian groups. II, Ann. of Math. (2) 91 (1971), 607-639.

[N] J. Noguchi, Moduli spaces of holomorphic mappings into hyperbolically imbedded complex spaces and locally symmetric spaces, Invent. Math. 93 (1988), 15-34.

[S] V. Savin, On moduli of Riemann surfaces, Soviet Math. Dokl. 12 (1971), 267-270.

[Sh1] H. Shiga, On analytic and geometric properties of Teichmüller spaces, J. Math. Kyoto Univ. 24 (1984), 441-452.

[Sh2] H. Shiga, Characterization of quasidisks and Teichmüller spaces, Tôhoku Math. J. 37 (1985), 541-552.

Depertment of Mathematics, Nagoya University, Chikusa-ku, Nagoya 464-01, Japan 